\title{
Advantages of polycarboxylic over dicarboxylic anhydrides in the melt modification of PPC
}

\author{
C. Barreto ${ }^{1,2}$, E. Hansen $^{1}$, S. Fredriksen ${ }^{2 *}$ \\ ${ }^{1}$ University of Oslo, Department of Chemistry Postboks 1033 Blindern, NO 0315 Oslo, Norway \\ ${ }^{2}$ Norner AS, Department of Polymer Research, Asdalstrand 291. NO 3960 Stathelle, Norway
}

Received 27 May 2013; accepted in revised form 14 July 2013

\begin{abstract}
We present an alternative polymer modification of poly (propylene carbonate) (PPC). A conventional practice for PPC melt processing is the melt - modification with maleic anhydride (MAH) - a di-carboxylic anhydride -. In our work, PPC is melt-modified with pyromellitic di-anhydride (PDAH) - a tetra-carboxylic dianhydride - . Using MAH modified PPC as reference, the polymer degradation and the thermal and viscoelastic properties of the materials are studied. Both anhydrides after their melt compounding have similar efficiency in the conservation of the molecular weight. Strikingly, the use of PDAH is advantageous over MAH considering the improvement of the thermal stability, the decrease of the complex melt viscosity and higher storage modulus (stiffness) of the modified PPC. It is speculated that the changes in the material performance arise from the occurrence of long chain branching and non-covalent interactions from the PDAH modifier.
\end{abstract}

Keywords: mechanical properties, thermal properties, pyromellitic dianhydride, polymer stability, poly(propylene carbonate)

\section{Introduction}

The transformation of carbon dioxide as a low cost monomer into useful materials like poly (alkylene carbonates) for packaging and consumer goods, has been globally proposed by industry and academia as a contribution to the mitigation of the greenhouse effect [1]. Poly(propylene carbonate) (PPC), the most studied of these polymers, faces multiple challenges when it comes to material properties and processing, in particular with respect to its mechanical properties, warpage and polymer degradation upon melting extrusion [2-5]. The esterification of the hydroxyl end groups of PPC using i.e. maleic anhydride (MAH), a dicarboxylic anhydride, has proven successful to mitigate the thermal degradation via chain unzipping and minimize the loss of molecular weight during extrusion [2-8]. Using anhydrides containing three or more carboxylic groups, like the tetra-carboxylic pyromellitic di- anhydride (PDAH), has however not been reported. In this short communication we want to report some advantages found for PDAH over MAH as melt modifiers of PPC, based on characterizations of the polymer stability, and the viscoelastic and thermal properties.

\section{Material and methods}

\subsection{Sample preparation}

PPC pellets (QPAC40 from Empower Materials, USA) (4.8\% propylene carbonate) were dried under dry $\mathrm{N}_{2}$ atmosphere at $25^{\circ} \mathrm{C}$ for $72 \mathrm{~h}$ prior to use. PDAH (Cat. \# 412287, $M_{\mathrm{W}} 218.32$, 97\%, Sigma Aldrich, Germany) and MAH (Cat. \# 63200, $M_{\mathrm{W}}$ 98.06, 99\%, Sigma Aldrich, Germany). PPC pellets $(300 \mathrm{~g})$ and anhydride powder $(0.1,0.5,1.0$ and $3.0 \mathrm{wt} \%$ ) was premixed and fed in a Prism Eurolab16 twin screw compounder (Thermo Electron Corporation, UK, Barrel $D: 16 \mathrm{~mm}, L / D: 25$, feed-

\footnotetext{
${ }^{*}$ Corresponding author, e-mail: siw.fredriksen@norner.no (C) BME-PT
} 
ing speed: $2 \mathrm{~kg} / \mathrm{h}$, screw speed: 500 r.p.m, barrel and die temperature: $140^{\circ} \mathrm{C}$ ). The extrudate was cooled by water, cut into pellets and dried under dry $\mathrm{N}_{2}$. The initial and final portions of the extrudate were discarded and approximately $120 \mathrm{~g}$ of each melt compounded recipe was collected.

\subsection{Characterization methods}

Rheology: Complex viscosity $\left(\eta^{*}\right)$, was measured within the linear viscoelastic range using the Rheometrics Scientific RDA II Analyzer, equipped with plate-plate geometry (diameter $25 \mathrm{~mm}$ ), on hot pressed specimens (diameter $25 \mathrm{~mm}$ ). Preheating time of $200 \mathrm{~s}$, frequency interval 0.06 to $15.85 \mathrm{rad} / \mathrm{s}$ for isothermal/isostrain measurements at $140^{\circ} \mathrm{C} / 10 \%$ was used.

TGA: Thermogravimetric analysis thermograms were recorded in a TGA Q500 instrument from TA Instruments in accordance to ISO 11358, using $3 \mathrm{~mm}$ diameter disks approximately of $10 \mathrm{mg}$, cut from plates $1 \mathrm{~mm}$ thick hot pressed at $150^{\circ} \mathrm{C}$. Constant flow of $\mathrm{N}_{2}$ was used during 5 min purging period and during the analysis. TGA runs were recorded over a temperature interval of $25-425^{\circ} \mathrm{C}$ using a heating rate of $10^{\circ} \mathrm{C} / \mathrm{min}$. The characterizations were made following the ICTAC guidelines for data acquisition [9].

GPC: The molecular-weight distribution was determined by size-exclusion chromatography (SEC). The analyses were performed in an Agilent PLGPC 50 equipped with a refractive index detector and calibrated with narrow polystyrene standards using THF as the eluent at $40^{\circ} \mathrm{C}$ (triplicate measurements).

DMTA: Dynamic mechanical thermal analysis characterizations were performed in a DMA Q800 from TA Instruments using a single-cantilever fixture. Storage and loss moduli, $E^{\prime}$ and $E^{\prime \prime}$, were measured within the linear viscoelastic range. The scanned interval was -5 to $70^{\circ} \mathrm{C}$ using a dynamic strain of $0.01 \%$ and a frequency of $1 \mathrm{~Hz}$; the test specimens were $2 \mathrm{~mm} \times 5 \mathrm{~mm} \times 25 \mathrm{~mm}$ strips cut from hot-pressed films at $150^{\circ} \mathrm{C}$ (triplicate measurements).

\section{Results and discussion}

The characterization of anhydride melt modified PPC (PPCMA: MAH modified, and PPCPA: PDAH

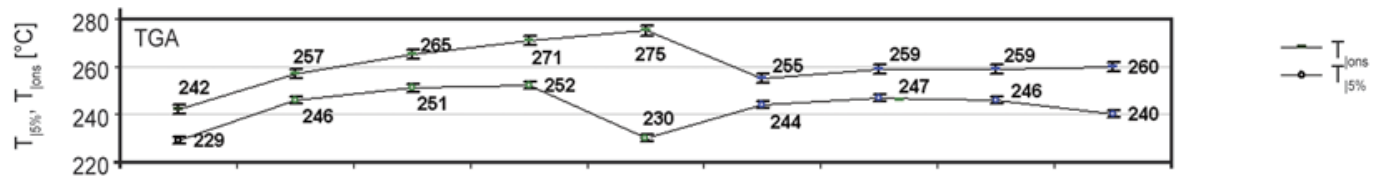

a)

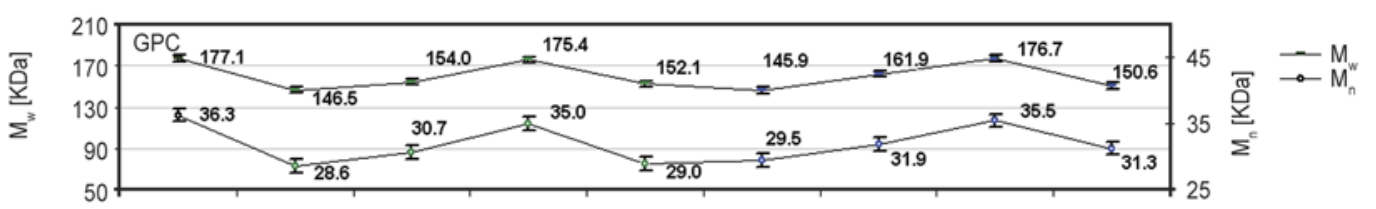

b)

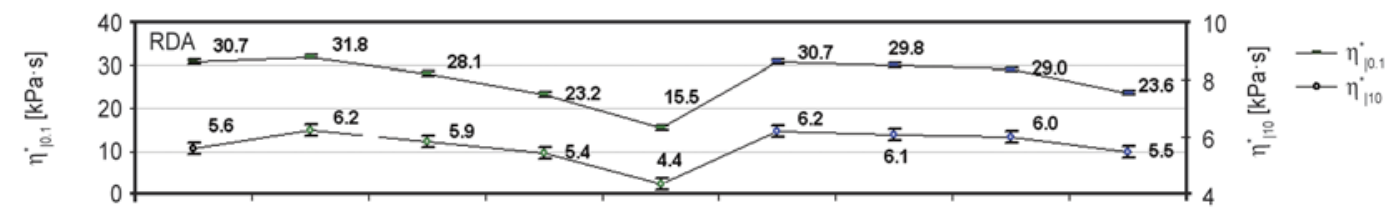

c)

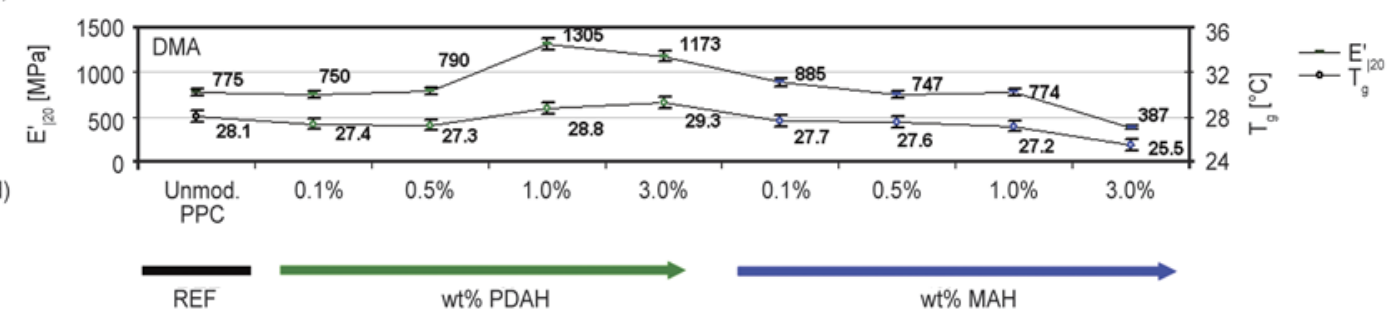

Figure 1. Characterization of unmodified PPC and melt modified PPC with maleic anhydride (MAH) and pyromellitic dianhydride $(\mathrm{PDAH})$ at various wt $\%$ loadings. a) TGA: $\mathrm{N}_{2} 10^{\circ} \mathrm{C} / \mathrm{min},\left.T\right|_{5 \%}\left[{ }^{\circ} \mathrm{C}\right]$ : temperature at $5 \mathrm{wt} \%$ loss, $T_{\text {ons }}\left[{ }^{\circ} \mathrm{C}\right]$ : temperature at the onset of the main thermal decomposition. b) GPC: $M_{\mathrm{n}}[\mathrm{kDa}]$ : molecular weight in number, $M_{\mathrm{w}}[\mathrm{kDa}]$ : molecular weight in weight. c) RDA: $\eta^{*} \mid 0.1$ and $\eta^{*}{ }_{\mid 10}[\mathrm{kPa} \cdot \mathrm{s}]$ : complex viscosity at 0.1 and $10 \mathrm{rad} / \mathrm{s}$. d) DMA: $T_{\mathrm{g}}\left[{ }^{\circ} \mathrm{C}\right]$ : glass transition temperature, $E_{\mid 20}^{\prime}[\mathrm{MPa}]$ : storage modulus at $20^{\circ} \mathrm{C}$ 
modified) by SEC, RDA, DMTA and RDA is shown in Figure 1. Reference samples are unmodified PPC (not extruded). The TGA parameters (Figure 1a) show the expected increase in the thermal stability for PPC after the addition of MAH and PDAH. At the highest level of anhydride addition ( $3 \mathrm{wt} \%), T_{55} \%$ decreases for both MAH and PDAH and $T_{\text {|ons }}$ increases steadily, clearly indicating the occurrence of degradation via chain unzipping (ChUz) probably caused by the excess of acidity in the system. This behavior at the $3 \mathrm{wt} \%$ level is consistent with the drop in the molecular weight $M_{\mathrm{n}}$ and $M_{\mathrm{w}}$ (Figure $1 b)$, the decrease of the complex viscosity $\left(\eta^{*} \mid 0.1\right.$ and $\left.\eta^{*}{ }_{10}\right)$ (Figure $\left.1 \mathrm{c}\right)$, and the stiffness $\left(E^{\prime}{ }_{20}\right.$ in Figure 1d).

The stoichiometric amounts required to complete the end capping in both polymer ends correspond approximately to $0.3 \mathrm{wt} \%$ loading for both anhydrides. Hence, a stoichiometric excess of acid equivalents are present in most of the samples. Further, it may be assumed that incomplete end capping happens in practice due to, e.g., short residence time and incomplete mixing in the compounder. Therefore, the improvement of properties approximately above $0.3 \mathrm{wt} \%$ anhydride are assumed to be explained also by non-covalent interactions between the unreacted anhydrides and the polymer chains or among unreacted anhydrides, e.g., hydrogen bonding and other non-covalent interactions (vide infra).

One of the major concerns regarding PPC compounding is the polymer degradation due to simultaneous thermal and mechanical (shear) degradation. Random chain scission (RCS) and $\mathrm{ChUz}$ of freshly generated hydroxyl chain ends is commonly evidenced in our work with end capped PACs. This shear dependent process affects in particular the high molecular weight fraction of the polymer as evidenced from the higher drop of $M_{\mathrm{w}}$ compared to $M_{\mathrm{n}}$ (Figure 1b). Both anhydrides act as chain extenders or chain healers as evidenced by the observed mitigation of the molecular weight decrease, similar to previously reported for MAH [7]. The chain healing process is depicted in Figure 2 and can be described as follows: during PPC extrusion in the absence of any anhydride, RCS occurs and new hydroxyl end groups are generated (Figure 2a). These end groups cause increase in the $\mathrm{ChUz}$ with the concomitant formation of cyclic propylene carbonate (CPC). In the presence of a di or tetra carboxylic compound like MAH or PDAH (Figure 2b$2 c$ ), the hydroxyl ends react with the carboxyl moieties in two ways. On the one hand, end capping occurs for the free polymer ends thus reducing $\mathrm{ChUz}$ in the conventional way. On the other hand, it is suggested that the anhydrides participate in a 'healing' reaction that remedies the molecular weight drop caused by the RCS. The chain 'healing efficiency' is similar for both anhydrides as seen from the similar values of $M_{\mathrm{n}}$ and $M_{\mathrm{w}}$ at the same level of anhydride. This fact suggests that the esterification rate for the two anhydrides with PPC is similar under the conditions used. The molecular weight conservation can be expected at slightly higher levels than $1 \mathrm{wt} \%$ of anhydride.

PPC containing CPC is soft since the latter has a plasticizing effect. The polymer softness leads to warpage and shrinkage of PPC items that have been processed by conventional polymer converting techniques like injection molding. Therefore, it is desirable to increase the polymer stiffness in order to

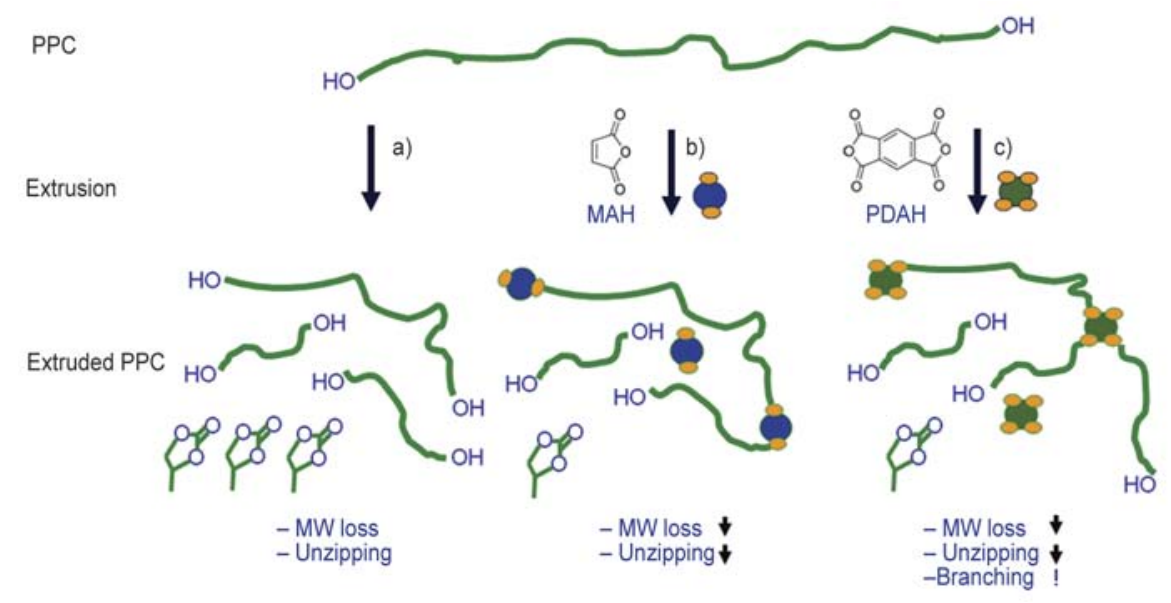

Figure 2. Hypothetical reaction paths during reactive extrusion in absence of additives a), in presence of dicarboxylic mono-anhydride (MAH) b) and tetracarboxylic di-anhydride (PDAH) c) 
counteract the relaxation of the built up internal stresses in order to mitigate these phenomena. The storage modulus $\left(E^{\prime}{ }_{20}\right)$, a measure of the stiffness, shows that PDAH contributes to increased stiffness (Figure 1d). The presence of more than two carboxylic groups in PDAH may allow the tethering of multiple PPC chains to a single dianhydride molecule (Figure 2c). Also, the slight modification of $T_{\mathrm{g}}$ that is observed follows the trend for $E_{\mid 20}^{\prime}$. It is worthwhile to mention that the resulting modified polymer samples are fully thermoplastic, and fully soluble in acetone which allows discarding the occurrence of strong crosslinking. Instead this behavior suggests that the improved mechanical properties may arise from branching from at least three PPC chains tethered to a PDAH molecule (Figure 2c). This hypothesis is also supported from previous work on chain extension in PLA in which the addition of polyfunctional chain extenders with isocyanide or epoxy moieties result in branched polymer structures $[10,11]$. Maleic anhydride reinforces the polymer at low loadings but softens the polymer at higher level. The drop in stiffness with increasing MAH loading is opposite to previous observations [7]. In our work it appears that the coupling of two maleate moieties which allows branching and explains the increase of stiffness in previous work [7] is a reaction of low significance. The changes in $E_{\mid 20}^{\prime}$ and $T_{\mathrm{g}}$ could also be understood as the result of a varying content of CPC in the samples. However, when evaluating the CPC content in the samples modified with $1.0 \mathrm{wt} \%$ of PDAH and MAH, both show insignificant differences in the CPC level. This observation allows discarding any significant effect of the CPC content in the changes in $E_{\mid 20}^{\prime}$ and $T_{\mathrm{g}}$ of samples modified with similar levels of both anhydrides under discussion. The characterization of $\mathrm{CPC}$ was done by ${ }^{1} \mathrm{H}-\mathrm{NMR}$ however, for brevity, was not included.

When checking the DMA and TGA characterization of samples, the dissimilar modification of the thermal stability and stiffness may also be explained from non-covalent interactions. Hydrogen bonding has been reported to contribute to both performances in PPC $[12,13]$. Also considering the presence of aromatic rings from PDAH, interactions with the $\pi$ electron cloud ( $\pi$-carbonyl, $\pi$-carboxyl or $\pi-\pi$ interactions [14]) could also be the origin of the improvements observed in these properties. The changes in $T_{\text {ons }}$ from TGA reveals the changes in the ability of the polymer to undergo degradation via $\mathrm{RChSc}$. The samples modified by PDAH show higher values in this parameter which is a sign of different levels of thermal stabilization as the result of the modification of non-covalent interactions.

The thermomechanical properties of PPC may also be enhanced by the compounding with organic and inorganic micro- and nanofillers. This commonly results in a steep increase of the melt viscosity, which affects the processing performance of the resulting compounds. A lower melt viscosity of the resin can be achieved by decreasing the molecular weight, but this is most often undesired. Preferably, the final molecular weight should be high while at the same time decreasing the melt viscosity to facilitate processing. This type of performance is achieved after the compounding of PPC with anhydrides, evidenced from the drop of the complex viscosity both at high and low shear (Figure 1c). Taking into account the mitigation of the molecular weight drop when increasing the anhydride loading (Figure 1b), the observed decrease of the complex viscosity can clearly not be linked to the decrease of the molecular weight. These facts allow speculating on the polymer structural reconformation and/or the modification of the secondary interactions with the anhydrides.

\section{Conclusions}

The melt modification with multifunctional carboxylic anhydrides like PDAH is a useful tool in high-shear melt processing of PPC. It is found that this alternative compared to the modification with MAH improves the stiffness, the thermal stability and decreases the melt viscosity of PPC while mitigates the molecular weight drop caused by shear. It is suggested that in addition to conventional endcapping, branching due to the tethering of more than two PPC chains to a single anhydride molecule, and the influence of non-covalent interactions may explain these results.

\section{Acknowledgements}

This work was funded with resources of the GASSMAKS program of the Research Council of Norway, and from Norner AS. The authors gratefully acknowledge the Scientific Laboratories at Norner AS for the sample characterizations. 


\section{References}

[1] Aresta M.: Carbon dioxide as chemical feedstock. Wiley, Weinheim (2010).

[2] Dixon D. D., Ford M. E., Mantell G. J.: Thermal stabilization of poly(alkylene carbonate)s. Journal of Polymer Science: Polymer Letters Edition, 18, 131-134 (1980).

DOI: $10.1002 /$ pol.1980.130180210

[3] Inoue S., Tsuruta T., Takada T., Miyazaki N., Kambe M., Takaoka T.: Synthesis and thermal degradation of carbon dioxide-epoxide copolymer. Applied Polymer Symposium, 26, 257-267 (1975).

[4] Luinstra G., Borchardt E.: Material properties of poly (propylene carbonates). Advances in Polymer Science, 245, 29-48 (2012).

DOI: $10.1007 / 122011126$

[5] Luinstra G. A.: Poly(propylene carbonate), old copolymers of propylene oxide and carbon dioxide with new interests: Catalysis and material properties. Polymer Reviews, 48, 192-219 (2008).

DOI: $10.1080 / 15583720701834240$

[6] Peng S., An Y., Chen C., Fei B., Zhuang Y., Dong L.: Thermal degradation kinetics of uncapped and endcapped poly(propylene carbonate). Polymer Degradation and Stability, 80, 141-147 (2003).

DOI: 10.1016/S0141-3910(02)00395-6

[7] Yao M., Mai F., Deng H., Ning N., Wang K., Fu Q.: Improved thermal stability and mechanical properties of poly(propylene carbonate) by reactive blending with maleic anhydride. Journal of Applied Polymer Science, 120, 3565-3573 (2011).

DOI: $10.1002 / a p p .33565$

[8] Zhang Z., Shi Q., Peng J., Song J., Chen Q., Yang J., Gong Y., Ji R., He X., Lee J-H.: Partial delamination of the organo-montmorillonite with surfactant containing hydroxyl groups in maleated poly(propylene carbonate). Polymer, 47, 8548-8555 (2006).

DOI: $10.1016 /$ j.polymer.2006.09.041
[9] Vyazovkin S., Burnham A. K., Criado J. M., PerezMaqueda L. A., Popescu C., Sbirrazzuoli N.: ICTAC Kinetics Committee recommendations for performing kinetic computations on thermal analysis data. Thermochimica Acta, 520, 1-19 (2011).

DOI: $10.1016 /$ j.tca.2011.03.034

[10] Corre Y-M., Maazouz A., Duchet J., Reignier J.: Batch foaming of chain extended PLA with supercritical $\mathrm{CO}_{2}$ : Influence of the rheological properties and the process parameters on the cellular structure. The Journal of Supercritical Fluids, 58, 177-188 (2011). DOI: 10.1016/j.supflu.2011.03.006

[11] Di Y., Iannace S., Di Maio E., Nicolais L.: Reactively modified poly(lactic acid): Properties and foam processing. Macromolecular Materials Engineering, 290, 1083-1090 (2005).

DOI: 10.1002/mame.200500115

[12] Chen L., Qin Y., Wang X., Li Y., Zhao X., Wang F.: Toughening of poly(propylene carbonate) by hyperbranched poly(ester-amide) via hydrogen bonding interaction. Polymer International, 60, 1697-1704 (2011). DOI: 10.1002/pi.3132

[13] Yu T., Zhou Y., Zhao Y., Liu K., Chen E., Wang D., Wang F.: Hydrogen-bonded thermostable liquid crystalline complex formed by biodegradable polymer and amphiphilic molecules. Macromolecules, 41, 31753180 (2008).

DOI: $10.1021 / \mathrm{ma} 7020562$

[14] Gautrot J. E., Hodge P., Cupertino D., Helliwell M.: Experimental evidence for carbonyl- $\pi$ electron cloud interactions. New Journal of Chemistry, 30, 18011807 (2006).

DOI: $10.1039 / \mathrm{b} 608628 \mathrm{~d}$ 